\title{
COVID-19 and geriatric clinical trials research
}

\author{
Elizabeth K. Rhodus ${ }^{1}$ (1) - Shoshana H. Bardach ${ }^{1,2}$ • Erin L. Abner ${ }^{1,3} \cdot$ Allison Gibson $^{1,4}$. Gregory A. Jicha $a^{1,5,6}$
}

Received: 4 June 2020 / Accepted: 31 August 2020 / Published online: 16 September 2020

(c) Springer Nature Switzerland AG 2020

Keywords COVID-19 $\cdot$ Research $\cdot$ Clinical Trails

\section{Introduction}

The SARS-CoV-2 pandemic has wrought tremendous upheaval to daily life, spreading rapidly and causing millions to contract COVID-19. The long-term effects are yet unknown, but scientists are studying the disease and searching for solutions. In geriatric clinical trials research, the problems presented by COVID-19 are multifaceted: older adults face increased risk of morbidity and mortality due to COVID-19, and so ongoing clinical trials that enroll geriatric participants have been disrupted, appropriately so, in light of these increased risks [1]. Yet, the interruption of ongoing trials may further increase risks to older adults as critical research on treatments for highly prevalent diseases and conditions is slowed or stopped. Further, older adults are substantially underrepresented in clinical trials research, and this situation may worsen this discrepancy. Scientists in aging appreciate the necessity of older adults' inclusion within clinical trials, but the vulnerability to increased exclusion in clinical trials of this population is high, now more than ever. Attention to clinical trial development with special attention to need for inclusion of older

Elizabeth K. Rhodus

Elizabeth.rhodus@uky.edu

1 Sanders-Brown Center on Aging, University of Kentucky, Lexington, KY 40536, USA

2 Graduate Center for Gerontology, University of Kentucky, Lexington, KY 40536, USA

3 Department of Epidemiology, University of Kentucky, Lexington, KY 40536, USA

4 College of Social Work, University of Kentucky, Lexington, KY 40536, USA

5 Department of Behavioral Science, University of Kentucky, Lexington, KY 40536, USA

6 Department of Neurology, University of Kentucky, Lexington, KY 40536, USA adults and precautions is greatly needed to sustain current efforts, at minimum, and ideally, enhance recognition of the value of including older adults in clinical trial research. The COVID-19 crisis affects every aspect of clinical trial research engagement including: recruitment and retention; ability to ensure participant safety while engaged in experimental interventions; study procedures, including consideration of remote assessments, impact on populations with health disparities, and generalizability of future results; outcome measures, including biomarker assessment; impact on the clinical trial workforce, including attrition; impact on dissemination of results and scientific collaborations, which move the clinical trial infrastructure forward; current and future funding allocations; and regulatory considerations in regards to management of altered study conduct and change of outcome measures (Fig. 1).

The purpose of this article is to highlight the impact of disasters such as the COVID-19 pandemic on geriatric clinical trials research and propose approaches for the scientific community to continue pushing forward.

\section{Impact on clinical trial operations}

Disruptions due to disaster can often result in disruptions to study protocols, challenges to data interpretation, the need for Institutional Review Board (IRB) amendments, and the introduction of new biases [2]. Prior research highlights the importance of proactive plans to minimize study disruption, the need to adapt rapidly to changing constraints, and the importance of ongoing communication with contract research organizations and sponsors and to share and develop strategies to mitigate participant-related protocol deviations and violations. Application of these actions should all occur in the context of local conditions.

Researchers have not previously seen this global level of disruption to clinical trials. Effects of COVID-19 on geriatric clinical trials research will be long-lasting. Trials in their 
Fig. 1 COVID-19 pandemic and other disasters impact every aspect of clinical trial engagement, conduct and outcomes

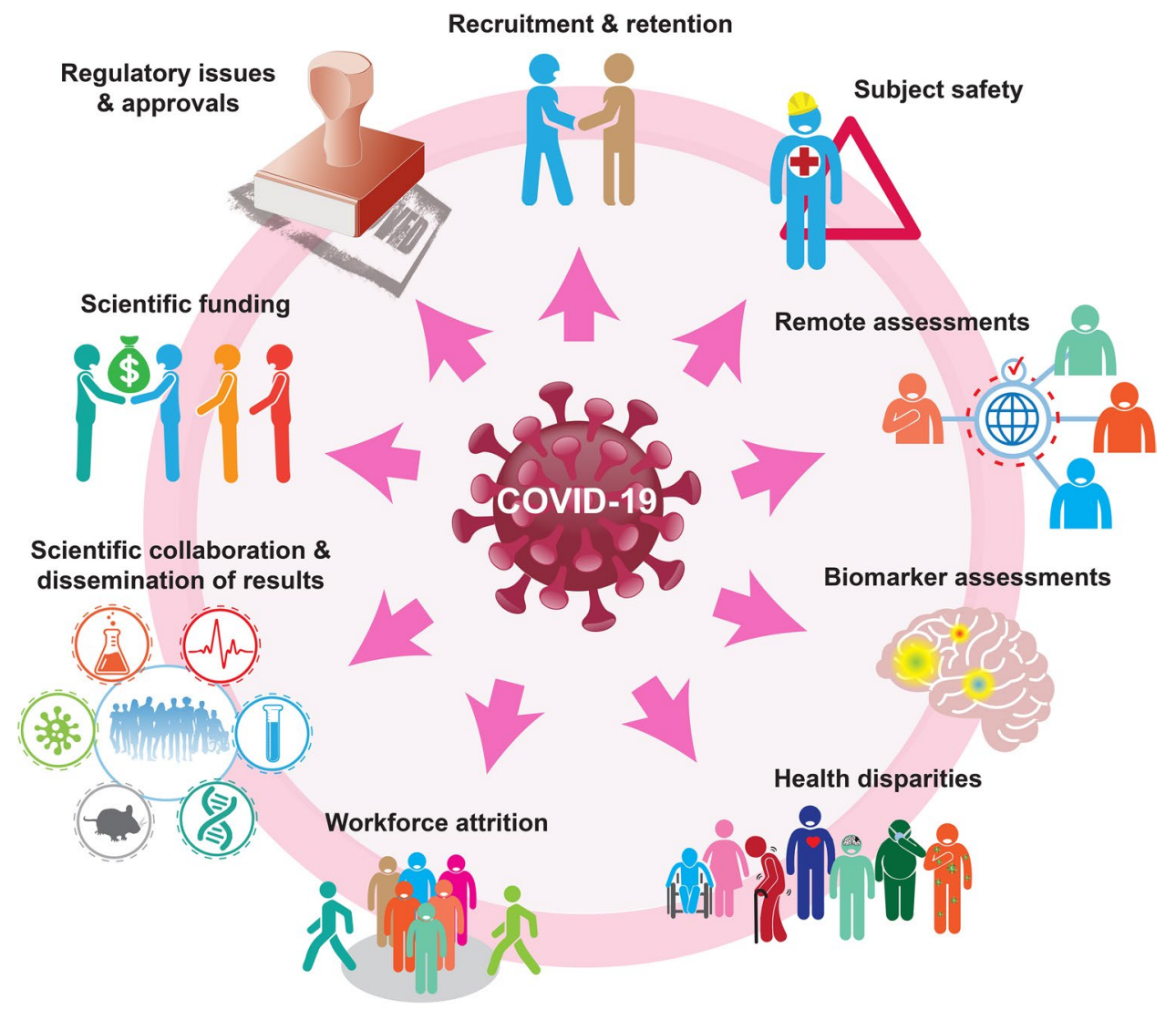

enrollment phase will at a minimum be delayed in achieving full recruitment, leading to lags in, or losses of, discovery. Depending on the duration of stay-at-home orders, some studies may be permanently disrupted and discontinued. For more established trials, if attrition leads to final sample sizes much smaller than those required by the a priori power estimates, then these ongoing studies may not have enough statistical power to identify positive findings. Trials that involve in-person cognitive assessment face challenges as they move to other modalities for testing, which may influence results. Efforts to validate existing measures through remote assessment should continue, to enable meaningful cognitive measures to be assessed remotely. In addition, investment in low cost technology to support these efforts, for participants who may not have remote access, may enable studies to continue with limited disruption, while also ensuring those of lower socioeconomic status are not left behind.

\section{Impact on participant safety and engagement}

Clinical trials involving those with cognitive impairment face unique challenges during such crises [1]. These individuals depend on routine for optimal functioning, as well as access to health care, and supportive community engagement. At a time of social distancing, routines and support systems are dramatically altered. Social cohesion and community can offer protections and resiliency for older adults during the time of disaster management. Maintaining and supporting active participant and recruitment engagement is essential. For example, the addition of virtual connection, training, and additional contact may prove highly beneficial.

Efforts to maintain engagement will need to be complemented by efforts to evaluate safety and efficacy. Many of the existing safety and efficacy measures within current trials are coming to a halt or being offered through non-traditional means, with unknown validity. Individuals continuing to take experimental medications may continue to have checkin safety calls but may have delays in bloodwork and imaging scans that would otherwise provide reassurance regarding any potential adverse medication effects. For infusion studies, medication delivery may be halted, but lingering effects of existing dosages are still possible. Careful evaluation of potential drug effects and critical safety measures will optimize the likelihood that safety is not compromised. Behavioral studies have additional challenges due to the prolonged proximity of participants and researchers required in some intervention. While the impact of abrupt halting of these trials is yet to be known, maintaining vigilance while planning to resume is essential. There is need for attention to implement gradual, careful restart processes that aim to keep participants safe while minimizing disruption to trials. 
These measures will maximize existing contributions and ensure the success of ongoing trials.

Assessing efficacy will be more complex, as the evaluation will necessitate consideration of history effects within studies including the upheaval to daily routines and changes in cognitive and social stimulation that COVID-19 has created. Methodological counterpoints will need to be considered including the role of randomization in distributing the psychosocial effects across groups, the timing of enrollment within the pandemic onset, and testing or controlling for psychological sequelae of COVID-19, such as controlling for depressive symptoms or the impact of social isolation and loneliness. Further, as noted above, changing test administration from in-person to digital modalities that can be conducted remotely out of the office will introduce additional variability into efficacy assessments.

\section{Disparate impact by age, race, and ethnicity}

In the US, morbidity and mortality early in the COVID-19 pandemic has disproportionately affected older adults and Black/African-American and Hispanic-American communities [3]. There are often perceptions in these communities, which arose through a long history of mistreatment by biomedical researchers and healthcare providers, that healthcare offers assistance but also potentially contributes to individual and community harm. If healthcare centers are seen as a focal point for contagion, engagement that requires in-person visits to healthcare facilities may be problematic. Existing barriers to engaging hard to reach participants will likely be exacerbated. Clinical discoveries should benefit everyone, and diversity and inclusiveness improve the generalizability of clinical trial findings. We must make concerted efforts in recruitment and retention to ensure advancements we have made in regard to increasing the representative population sample engaged in clinical trial research are sustained.

\section{Impact on biomarker outcomes}

Recent past and anticipated future successes in clinical trial activity in the area of aging and dementia have relied on biomarker assessments including: brain imaging (MRI, PET and other), which requires shared and repetitive usage of common acquisition facilities; retinal imaging and EEG, which require the use of shared equipment that may be difficult to sterilize or sanitize between participants. Biomarker outcomes are an increasingly important part of clinical trial research [4]. Discovery of disease-modifying therapies in the area of dementia research, especially preclinical dementia, is dependent on such measures. Coordination of using shared medical facilities such as imaging equipment including MRI and PET scanners for continued conduct has been a challenge due to concerns of COVID-19 transmission, despite the critical need for the acquisition of essential imaging and safety information mandated by participant status and clinical trial conduct. Development of specific and diligent methods to sterilize, sanitize, and convey these safety precautions to the public will allow continued engagement in clinical research activities.

\section{Impact on clinical trials workforce}

As with prior disasters, many research sites in the US have been forced to shut down and/or limit activities as a result of local or state restrictions due to COVID-19. In addition, site staff in many areas of the US are under 'remote work' or 'stay at home' orders that limit access to their workspace. Thus, critical research activity that supports the financial infrastructure of these research sites may be diminished and/ or completely disappear. It may not be possible for some investigators to continue to provide salary support for their well-trained research staff members, not only leading to dissolution of the existing research site infrastructure but also contributing further to the ongoing societal burden of the COVID-19 crisis. Such impact may also impede advancement of researchers' careers.

The need for the cultivation and maintenance of a workforce trained for enhancing older adults' engagement in clinical trial activities has been overlooked for years. Hiring and retaining qualified and experienced study coordinators, recruiters, psychometrists, and even phlebotomists in the geriatric research setting can be extremely difficult. Developing such skill sets and desires to work in research may be severely limited by the COVID-19 crisis unless we are able to ensure stability of the underlying research infrastructure. Otherwise, we will lose many preciously talented individuals who have entered the field of geriatric clinical research. The call for advocacy is needed for sustained funding mechanisms that offer emergency support and stable institutional standing to ensure continuity and protection of these positions.

\section{Immediate and future funding allocation}

Given the heightened public interest in advancing scientific and medical initiatives to combat the COVID-19 crisis, as well as the financial burden the crisis is posing globally, there is increasing competition for resources that have long been lacking in the field. Funding increases for agingrelated research were moving at a rapid pace but funds now may be in jeopardy with this competing need. Large-scale funding from the NIH is being redirected to directly combat the COVID-19 pandemic. While immediate repercussions on the budget for National Institute on Aging has not yet occurred, we hope that specific aging-related research continues to receive the funds necessary to move the field 
forward. Representation and advocacy for aging science by stakeholders is imperative to protect current availability of geriatric clinical trial research.

\section{Impact on future research}

As scientists consider lasting implications of COVID-19, future study design planning and management will likely be altered. Specifically, increased utilization of participantcentered design while maintaining rigor and reproducibility is crucial. Study design that promotes engagement in a way that meets older adults' unique needs with enhanced ease in access will be needed. Current and ongoing recruitment avenues and disaster preparedness plans must acknowledge ways to mitigate decreased contact with older participants who do not use email or computers. Further, care for participants that typically occurs during clinical trials under the supervision of medical professionals will need to be adjusted to maintain safety.

Development, maintenance, and implementation of disaster and business continuity plans within clinical trial design is needed in future planning. Mische and Wilkerson [5] describe the need for preparation using risk analyses, response during disasters with implementation of essential functions, recovery efforts, and mitigation of losses to clinical trial data and resources. Clinical trial continuity for interventions and care of older adults, especially those with cognitive impairment, is paramount. The vulnerability of older adults to COVID-19 is a critical reminder for the need to prepare for disasters during clinical trial design.

Future changes in regulatory and industry perspectives on trial conduct could be profound. New trial design methodologies will need to be developed to take into account significant periods of time for subpopulation and/or total population of subjects that may not be able to conduct regular research activities. Our patient population regularly encounters unexpected healthcare, social, and/or personal crises that are considered significant disruptions to smooth research conduct. The potential for increasing recognition of such confounds in interpreting clinical trial data could help to smooth regulatory pathways for studies that encounter such obstacles in the future.

\section{Conclusion}

Scientists face unprecedented challenges with long-lasting impacts due to COVID-19. By learning from prior disasters and epidemics, we can be prepared to address current and upcoming changes. The resilience and ability of researchers to move science forward for the success of advanced care for older adults will have benefits for decades to come. Increased acknowledgement of a variety of social determinants of health will allow improved efforts to ensure innovative analytic approaches, as well as structures to support the research workforce and opportunities that keep research dissemination and scientific discovery active and productive.

Authors' contribution All authors contributed to the design, writing, and revision of the resulting manuscript. Authors thank Matt Hazzard and Tom Dolan, Medical Illustrators, University of Kentucky-College of Medicine for visual contributions.

Funding The first author is funded by NIH T32 AG057461: "Training in Translational Research in Alzheimer's and Related Dementias (TRIAD)". All authors are affiliated with University of Kentucky Alzheimer's Disease Cohort participants funded by NIH/NIA P30 AG028383.

Availability of data and materials Availability of data and material is not applicable.

\section{Compliance with ethical standards}

Conflict of interest The authors declare that they have no conflict of interest.

Statement of human and animal rights This article does not contain any studies with human participants or animals performed by any of the authors.

Informed consent For this type of article, formal consent is not required.

\section{References}

1. Nanda A, Vura N, Gravenstein S (2020) COVID-19 in older adults. Aging Clin Exp Res. https://doi.org/10.1007/s40520-02001581-5

2. Cherry KE, Calamia MR, Elliott EM (2020) When disasters strike: Navigating the challenges of "sudden science". In: Cherry KE, Gibson A (ed) The intersection of trauma and disaster behavioral health. Springer International Publishing. https://www.springer. com/us/book/9783030515249

3. Mays J, Newman A (2020) Virus is twice as deadly for black and Latino people than whites in N.Y.C., in The New York Times. The New York Times Company

4. Cummings J (2019) The National Institute on aging-Alzheimer's Association framework on Alzheimer's disease: application to clinical trials. Alzheimer's Dementia 15:172-178

5. Mische S, Wilkerson A (2016) Disaster and contingency planning for scientific shared resource cores. J Biomol Tech 27:4-17

Publisher's Note Springer Nature remains neutral with regard to jurisdictional claims in published maps and institutional affiliations. 